\title{
Erratum \\ Erratum to: Existence and Regularity of Very Weak Solutions of the Stationary Navier-Stokes Equations
}

\author{
HYUNSEOK KIM
}

\author{
Communicated by C. LE BRIS
}

\section{Erratum to: Arch. Rational Mech. Anal. (2009) 193:117-152 DOI 10.1007/s00205-008-0168-7}

The purpose of this erratum is to correct some mistakes of Remark 1, Lemma 1 and their consequences in the paper [Arch Rational Mech Anal 193 (2009) 117-152].

The major contribution of the paper is to establish existence and regularity of very weak solutions in $L^{q}(\Omega)$ of the Dirichlet problem (NS) for the stationary Navier-Stokes equations with arbitrarily large boundary data $g$ in $W^{-1 / q, q}(\partial \Omega)$. In particular, it is shown in Theorem 1 , the main existence result, that there exists at least one very weak solution $u \in L^{q_{0}}(\Omega)$ of (NS) in the sense of Definition 1 for the data $f, k$ and $g$ satisfying the very weak regularity (1). The proof of Theorem 1 is complete. Theorems 2 and 3, the main regularity results, are also correct. So are the corresponding results for the linear problems, i.e., Theorems 7, 9 and 10. However, due to the very weak regularity of $f$ and $k$, the solution $u$ neither satisfies (NS) in the usual distribution sense, nor does it have a well-defined trace, which contradicts the assertions in Remarks 2 and 3. In fact, Remarks 2 and 3 are wrong and should be corrected by imposing a stronger regularity assumption on $f$ and $k$.

Let $X$ and $Y$ be Banach spaces. Assume that $X \hookrightarrow Y$, i.e., $X \subset Y$, and that there is a constant $C>0$ such that $\|x\|_{Y} \leqq C\|x\|_{X}$ for all $x \in X$. Then the restriction of each $f \in Y^{\prime}$ to $X$, denoted $\left.f\right|_{X}$, is a bounded linear functional on $X^{\prime}$ with the norm bounded by $C\|f\|_{Y^{\prime}}$. Consider the space $\left.Y^{\prime}\right|_{X}=\left\{\left.f\right|_{X}: f \in Y^{\prime}\right\}$ equipped with the quotient norm

$$
\|\hat{f}\|_{\left.Y^{\prime}\right|_{X}}=\inf \left\{\|f\|_{Y^{\prime}}: f \in Y^{\prime}, \hat{f}=\left.f\right|_{X}\right\} \quad\left(\left.\hat{f} \in Y^{\prime}\right|_{X}\right) .
$$

It is easy to show that $\left.Y^{\prime}\right|_{X} \hookrightarrow X^{\prime}$. Moreover, if $X$ is dense in $Y$, then the restriction map $\left.f \in Y^{\prime} \mapsto f\right|_{X}$ is one-to-one and indeed an isometry from $Y^{\prime}$ onto $\left.Y^{\prime}\right|_{X}$, which

The online version of the original article can be found under doi:10.1007/s00205-0080168-7. 
verifies $Y^{\prime} \hookrightarrow X^{\prime}$. However, this embedding result fails to hold in cases when $X$ is not dense in $Y$. Therefore, Remark 1 is completely wrong and should be deleted; obviously, $W_{0}^{2, q^{\prime}}(\Omega)\left(W_{0}^{1, q^{\prime}}(\Omega)\right.$, respectively) is not dense in $W_{0}^{1, q^{\prime}}(\Omega) \cap W^{2, q^{\prime}}(\Omega)$ $\left(W^{1, q^{\prime}}(\Omega)\right.$, respectively).

Many of the results in the paper are based on the validity of Remark 1 and thus statement of these results should be corrected as follows:

(i) In the vector equations in Remarks 2 and $9, f$ should be changed by $\left.f\right|_{W_{0}^{2, q^{\prime}}(\Omega)}$, the restriction of $f$ to $W_{0}^{2, q^{\prime}}(\Omega)$. To identify $\left.f\right|_{W_{0}^{2, q^{\prime}}(\Omega)}$ with $f$ itself, we need to impose a stronger regularity assumption on $f$. In view of the Sobolev embedding theorem (see Remark 4), it suffices to assume that $1<r \leqq q<\infty, \frac{1}{n}+\frac{1}{q} \geqq \frac{1}{r}$ and $f \in W^{-1, r}(\Omega)$. More generally, we may assume that $f \in Y^{\prime}$, where $Y$ is any Banach space such that $W_{0}^{1, q^{\prime}}(\Omega) \cap W^{2, q^{\prime}}(\Omega) \hookrightarrow Y$ and $W_{0}^{2, q^{\prime}}(\Omega)$ is dense in $Y{ }^{1}$

(ii) The hypothesis for the trace theorem, Theorem 5 , (and similarly for Theorem 8) should be changed by $1<r \leqq q<\infty, \frac{1}{n}+\frac{1}{q} \geqq \frac{1}{r}, v \in L^{q}(\Omega)$, $p \in W^{-1, q}(\Omega),-\Delta v+\nabla p \in W^{-1, r}(\Omega)$ and $\operatorname{div} v \in L^{r}(\Omega)$.

(iii) The hypotheses for Remark 3 and Theorem 6 should be changed by $1<r \leqq$ $q<\infty, \frac{1}{n}+\frac{1}{q} \geqq \frac{1}{r}, f \in W^{-1, r}(\Omega), k \in L^{r}(\Omega)$ and $g \in W^{-1 / q, q}(\Omega)$.

(iv) Remark 12 should be corrected. Theorem 7 allows one to deduce the existence of a vector field $v \in L^{q}(\Omega)$ such that (9) holds for all $\varphi \in W^{1, q^{\prime}}(\Omega)$ and $\|v\|_{L^{q}(\Omega)} \leqq C\|(0, k, g)\|_{-2, q}$. If $1<r \leqq q<\infty, \frac{1}{n}+\frac{1}{q} \geqq \frac{1}{r}$ and $k \in L^{r}(\Omega)$, then we can choose $v$ so that $\operatorname{div} v=k$ in $L^{r}(\Omega)$ and $\gamma v=g$ in $W^{-1 / q, q}(\partial \Omega)$ as well.

Lemma 1 and its consequences should be also corrected as follows. In the proof of Lemma $1, u \in L^{q_{0}}(\Omega)$ is decomposed into $u=u_{S}+u_{r}$ with $C\left\|u_{S}\right\|_{L^{q_{0}(\Omega)}} \leqq \varepsilon$ and $\left\|u_{r}\right\|_{L^{\infty}(\Omega)} \leqq C_{\varepsilon}\|u\|_{L^{q_{0}(\Omega)}}$. In general, however, $C_{\varepsilon}$ depends on $u$. Hence the constant in Lemma 1 (ii) should depend on $u$ itself as well as its norm. Therefore, the constants in Lemmas 4-6 and Propositions 1-3 should depend on the given fields $u_{1}$ and $u_{2}$.

Acknowledgments. The author would like to thank Professor Chérif Amrouche for pointing out the mistake in Remark 1 with a clear counterexample.

Department of Mathematics, Sogang University, Seoul 121-742, Korea. e-mail: kimh@sogang.ac.kr

Published online April 13, 2010 - (C) Springer-Verlag (2010)

1 This is studied in more detail by C. Amrouche and M.A. Rodríguez-Bellido [Stokes, Oseen and Navier-Stokes equations with singular data, preprint]. 